\title{
A Satellite Relative Navigation based on Hardware Characteristics of Femtosecond Laser
}

\author{
Dae-Eun Kang, Sang-Young Park, Jongwoo Lee \\ Astrodynamics and Control Lab., Department of Astronomy, Yonsei University \\ 03722, Seoul, Korea \\ rkdeodms@gmail.com; spark624@yonsei.ac.kr; jwlee@galaxy.yonsei.ac.kr
}

\begin{abstract}
This study presents a satellite relative navigation system based on using a femtosecond laser distance meter. The satellite relative navigation system includes some hardware properties of an actual laser instrument in order to estimate relative distance between satellites in formation flying. To obtain the laser distance data, hardware parameters of a laser distance meter are updated and noises from elements of the laser instrument are added. Relative navigation simulation utilizing the updated measurement data is conducted, and the consequent results are compared with those from simulation with existing measurement data. In addition, a relationship is provided that is about lens radius, laser power and a limit distance defined to represent performance of the distance meter. With this relationship, we can acquire a relative distance range appropriate to use a femtosecond laser distance meter for space missions in formation flying.
\end{abstract}

Keywords: relative navigation test-bed, miniaturized femtosecond laser, formation flying, hardware properties

\section{Introduction}

Formation flying with satellites offers many advantages in space missions. There are various satellite formation flying missions using satellite relative navigation, such as GRACE [1], PRISMA [2], and JC2Sat-FF [3]. The current study concerns relative navigation between satellites in formation flying. The final goals of this study are improving a real-time satellite relative navigation algorithm using a femtosecond laser instrument and creating a blueprint for mounting it on satellites in formation flying.

There are numerous previous studies for relative navigation utilizing a femtosecond laser. A hardware-in-the-loop simulator (HILS) was designed as a test-bed to simulate space missions with GPS signal and laser ranging data [4-5]. A relative navigation algorithm based on laser ranging was developed [6]. Recently, a laser-based relative navigation algorithm using GPS measurements [7] and an improved GPS-based algorithm using laser distance measurements [8] have been developed. These previous studies could not adopt much information about real femtosecond laser instrument hardware, because they predated completion of such a hardware system. Thus, the current study seeks to improve the HILS by making the laser measurement data closer to the actual hardware data of a femtosecond laser. Satellite relative navigation is performed by a software-based simulator using hardware characteristics of a femtosecond laser. The femtosecond laser data are generated from a basic laser ranging theory and parameters are set based on conceptual design. The parameters in the simulator are the values of an actual instrument, and data noises based on the properties of the instrument. The results of relative navigation using the updated measurement data are analyzed and compared with those from simulations in previous studies. Furthermore, this study provides an equation to calculate the appropriate specification of a femtosecond laser instrument for relative navigation in space missions. Use of this relationship can yield a range of relative distance appropriate for a femtosecond laser system used in satellite relative navigation.

\section{Revision of Femtosecond Laser Measurement Data}

\subsection{Laser Measurement Data}

The minimized femtosecond laser distance meter and measurement data simulator utilized in this study are produced by Laboratory for Precision Engineering and Metrology (PEM), Korea Advanced Institute of Science and Technology 
(KAIST). The femtosecond laser distance meter uses the frequency comb of a femtosecond laser. The laser consists of several frequencies, which makes phase data in several ranges. An ambiguity is decided by differences in long waves due to slight changes of the laser frequency. After the integer ambiguity decision, distance is calculated more precisely by using a short wave [9].

Figure 1 shows layout of the femtosecond laser distance meter, which is composed of a laser signal generation part and a measurement data generation part. A laser simulator equipped with developed software embodies the two parts to generate measured distance data. First, the laser signal generation part makes laser signals according to distance between satellites. The measurement data generation part decides an integer ambiguity and distance data with the created laser signals. The laser signal generation part has many parameters, such as wave length, laser power, radius of beam, radius of lens, intensity-signal ratio of photo detectors, and ratios of long or short wave signals to a total signal amplitude. The other part has two parameters, a noise magnitude of photo detector and a resolution of phase meter. The parameters determined by previous studies are revised to those of the actual femtosecond laser instrument shown in Table 1. Because the longwave parameters are not concerned with precision, they have been left unchanged. The parameters of the measurement data generation part also have been preserved because their specifications have not changed. Actual measurement data includes some measurement noises which are not reflected in the software-based simulator. Because individual properties of elements of the instrument cause accumulated noises and the accumulation is too complicated to be expressed functionally, the noise level is acquired empirically by measuring errors at a fixed distance of $1 \mathrm{~m}$ for 1000 seconds. Figure 2 shows the standard deviation of the hardware noises is $109.935 \mu \mathrm{m}$. Hardware noises due to elements comprising the instrument are added to the measurement characteristics. Figure 3 shows errors of measurement data with initial and updated setting. An offset in mean of errors disappears and standard deviation of the measurement is increased after revisions. In addition, the hardware noises with $109.935 \mu \mathrm{m}$ are dominant in terms of precision up to about $10 \mathrm{~km}$ in the result with updated settings.

Table 1: Change of parameters in the laser distance measurement simulator.

\begin{tabular}{|l|c|c|}
\hline & $\begin{array}{c}\text { Existing values } \\
\text { used in previous studies }\end{array}$ & $\begin{array}{c}\text { Revised values } \\
\text { used in the current study }\end{array}$ \\
\hline Short wavelength [m] & 0.01 & 0.292141 \\
\hline Long wavelength [m] & 10 & 10 \\
\hline Laser power [W] & 1 & 0.01 \\
\hline Beam radius [m] & 0.1 & 0.001745 \\
\hline Lens radius [m] & 0.1 & 0.0254 \\
\hline Intensity-signal ratio [V/W] & 30 & 500 \\
\hline Short wave signal ratio & 0.00026667 & 0.1 \\
\hline
\end{tabular}

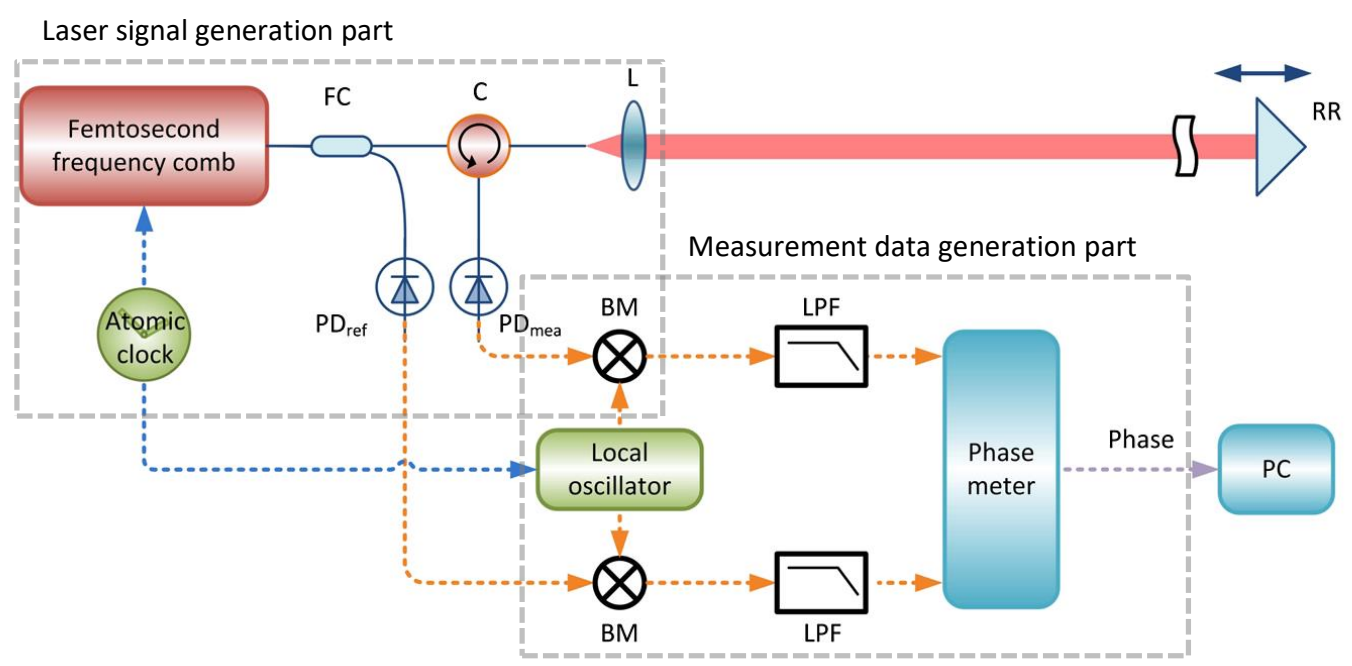

Fig. 1: Femtosecond laser instrument layout [9]. 


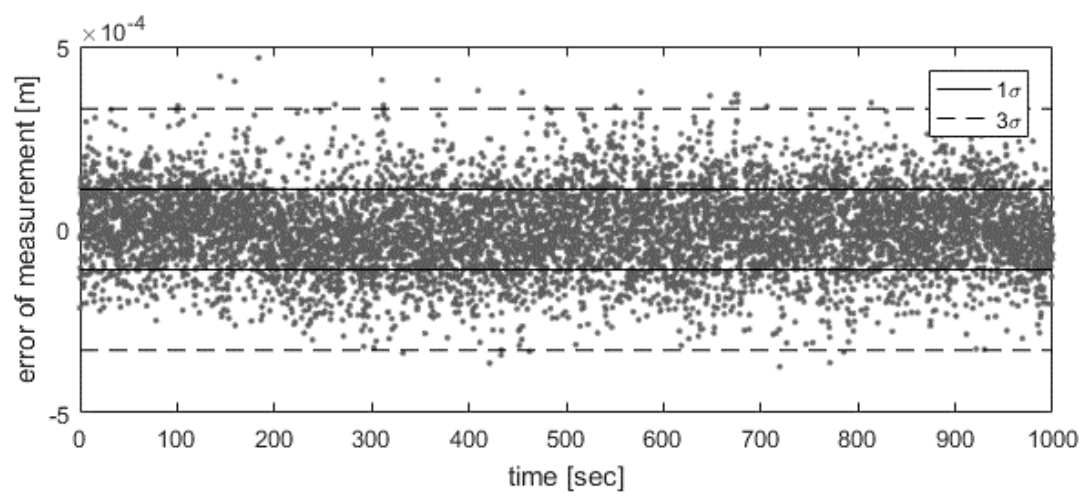

Fig. 2: Measurement error of the femtosecond laser distance meter on $1 \mathrm{~m}$ experiment.
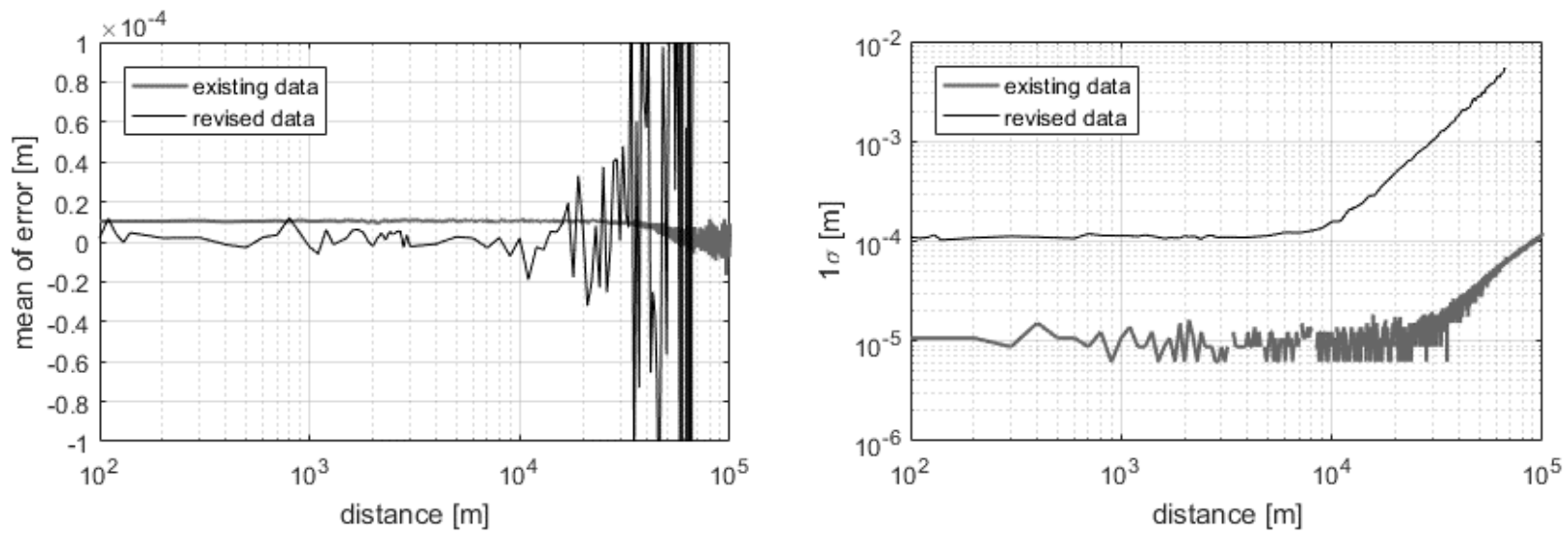

Fig. 3: Mean and standard deviation of laser measurement data errors over distance.

\subsection{Relative Navigation Result using Revised Measurement Data}

Relative navigation simulation is conducted with the revised laser measurement data. The simulation adopts the laserbased relative navigation algorithm of Jung et al. [6]. The orbit is set with an initial relative distance of $250 \mathrm{~m}$. The initial position and velocity of satellites are given by $r_{1}=\left[\begin{array}{llll}6930.00 & 0 & 0\end{array}\right] \mathrm{km}, v_{1}=\left[\begin{array}{llll}0 & 3.81095 & 6.60075\end{array}\right] \mathrm{km} / \mathrm{s}, r_{2}=$ $\left[\begin{array}{lll}6930.25 & 0 & 0\end{array}\right] \mathrm{km}, v_{2}=\left[\begin{array}{lll}0 & 3.81082 & 6.60053\end{array}\right] \mathrm{km} / \mathrm{s}$ in the Earth Centered Inertial coordinate.
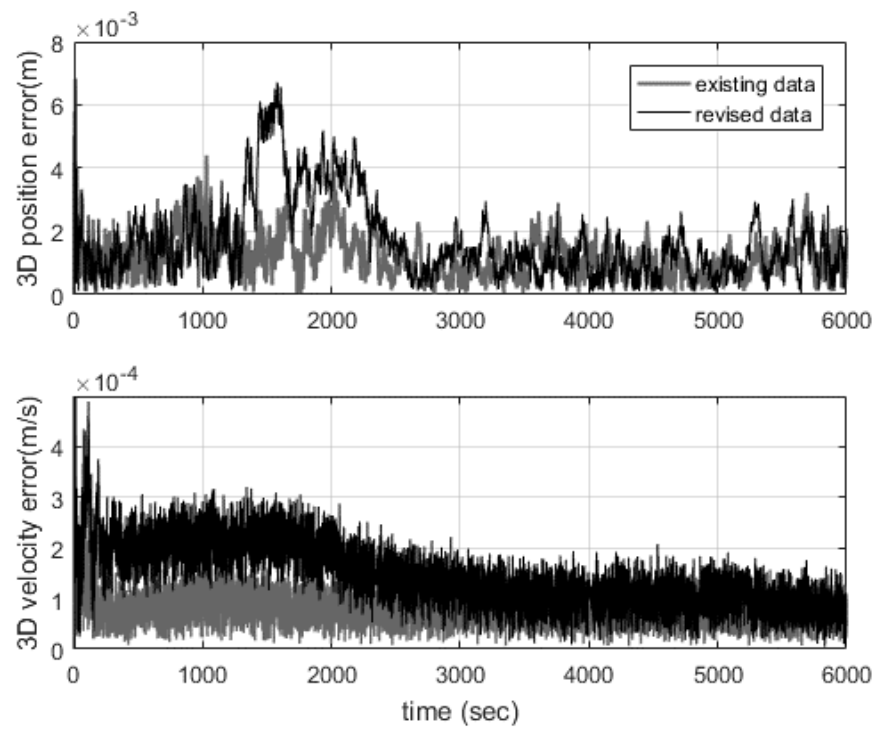

Fig. 4: RMS of position \& velocity errors of relative navigation simulation. 
Figure 4 shows the root mean square (RMS) values for errors of relative position and velocity in three-dimensional space. Grey lines are results using existing laser data and black lines are results using the revised data. By comparing the results of updated simulation to those of the existing one, the averages of errors in position and velocity increase from 1.37 $\mathrm{mm}$ and $86 \mu \mathrm{m} / \mathrm{s}$ to $2.16 \mathrm{~mm}$ and $152 \mu \mathrm{m} / \mathrm{s}$, respectively. They increased by $0.79 \mathrm{~mm}$ and $66 \mu \mathrm{m} / \mathrm{s}$ compared to the result using existing measurement data. The femtosecond laser distance meter is less precise than the initial design because of the unexpected hardware noises. However, the simulation results are closer to a practical mission environment by applying the hardware properties of the actual instrument.

\section{Relationship between Specifications and Performance}

The standard deviation of data errors increases from about $10 \mathrm{~km}$ in Figure 3. Measurement data lacks sufficient precision when distance between satellites is longer than $10 \mathrm{~km}$, which is a limit distance for utilizing the femtosecond laser distance meter. To extend the limit distance, specification of elements in the instrument should be improved. This study observes a change of the limit distance by modulating simulator parameters associated with instrument size, laser power, and lens radius, which is represent a size of optical elements in the interferometer of the femtosecond laser.

\subsection{Limit Distance}

Simulations are performed to obtain measurement errors over distance. The laser power and lens radius in the simulations are set to 0.01-0.2 W and 0.0254-0.07 m, respectively. Ranges where $3 \sigma$ of error is below $1 \mathrm{~mm}$ are defined as observable range, and then limit distance is the maximum distance in the range with an error under $1 \mathrm{~mm}$. The upper limit, $1 \mathrm{~mm}$, which implies that the instrument has sub-millimeter precision, is decided arbitrarily. The upper limit should be redecided in accordance with specific mission requirements. Figure 5 shows that the limit distance increases with growth of parameters. The precision in the observable range is preserved regardless of the distance and other parameters. To find the relationship between the laser power, the lens radius and the limit distance, a graph is drawn and fitted (Figure 6).

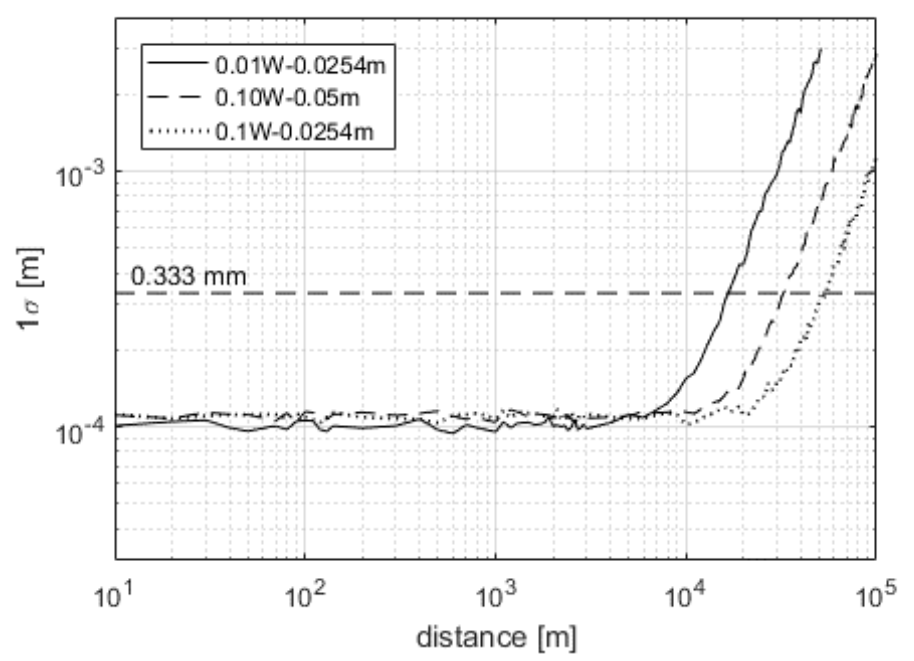

Fig. 5: Standard deviation of laser simulation errors over distance. 


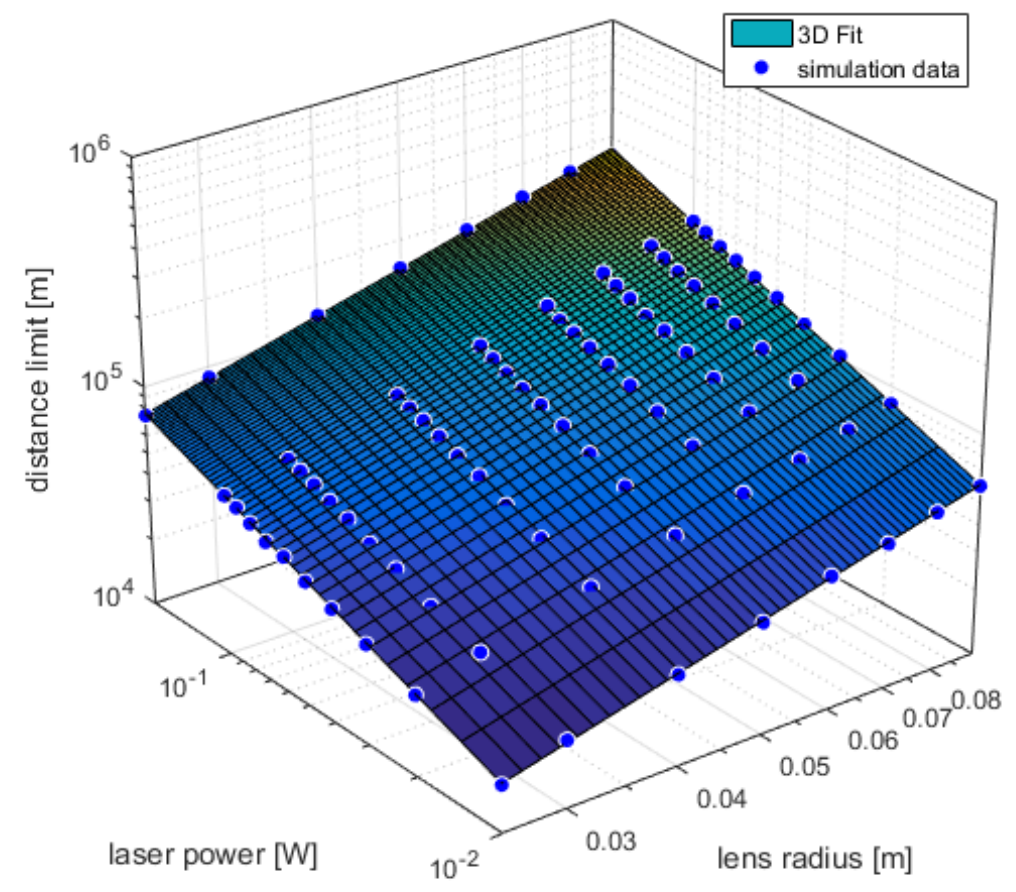

Fig. 6: Plane of the laser power, the lens radius and distance limit. Blue dots are data and grid plane is fitting result.

\subsection{Fitting Result}

The relationship between the lens radius, the laser power and the limit distance forms a plane in a logarithmic scale with blue dots in Figure 6. If each parameter is set to $X[\mathrm{~m}], \mathrm{Y}[\mathrm{W}], \mathrm{Z}[\mathrm{m}]$ in sequence, Eq. (1) expresses the relationship between them.

$$
\mathrm{c}_{1} \log X+\mathrm{c}_{2} \log Y+\mathrm{c}_{3} \log Z+\mathrm{c}_{4}=0
$$

where $c_{1}-c_{4}$ are arbitrary coefficients. Eq. (2) shows fitting result using Eq. (1). The coefficients, $p_{1}-p_{3}$, have $95 \%$ confidence bounds.

$$
\begin{gathered}
Z=X^{p_{1}} \cdot Y^{p_{2}} \cdot p_{3} \\
p_{1}=1, p_{2}=0.5, p_{3}=6.631 \mathrm{e} 6\left[W^{-\frac{1}{2}}\right]
\end{gathered}
$$

The grid plane in Figure 6 is the fitting result. The limit distance calculated by substituting the parameters of the actual instrument into Eq. (2) is $16.841 \mathrm{~km}$, and it has a difference of about $0.249 \mathrm{~km}$ from the limit distance of the simulation, $16.593 \mathrm{~km}$. By using Eq. (2), the maximum for the satellite relative navigation using the femtosecond laser distance meter can be found. On the contrary, required specifications of the distance meter also can be found for the satellite relative navigations.

\section{Conclusions}

The laser measurement simulator in the test-bed is updated by adapting the actual hardware properties of a femtosecond laser. Satellite relative navigation simulations are conducted with the updated measurement data from the laser instruments. The relative navigation results indicate that the test-bed becomes less precise but more practical than before. Furthermore, by changing the key parameters that influence the laser measurement result, standard deviation of the laser data error is observed to find a relationship between them. As a result, the ability of the femtosecond laser in the observable range is preserved irrespective of distance and some specifications of distance meter. We also provided the 
equation representing the relationship between the limit distance, the lens radius and the laser power. Specifications of the laser distance meter can be calculated from this equation to find appropriate distances for space relative navigations using the femtosecond laser. In the next study, we will find a complete equation including other parameters omitted in this study. Hardware properties will be also studied after a beam stabilization system is combined.

\section{Acknowledgements}

This work was supported by a Global Surveillance Research Center (GSRC) program funded by the Defense Acquisition Program Administration (DAPA) and Agency for Defense Development (ADD).

\section{References}

[1] B. D. Tapley, S. Bettadpur, J. C. Ries, P. F. Thompson and M. M. Watkins, "GRACE measurements of mass variability in the Earth system," Science, vol. 305, no. 5683, pp. 503-505, 2004.

[2] S. D'Amico, J.-S. Ardaens, and S. D. Florio, "Autonomous formation flying based on GPS - PRISMA flight results," Acta Astronaut., vol. 82, no. 1, pp. 69-79, 2013.

[3] A. de Ruiter, J. Lee, A. Ng, and Y. Kim, "Orbit Determination \& Relative Positioning Techniques for JC2Sat-FF," in Proceedings of the $3^{\text {rd }}$ International Symposium on Formation Flying, Missions and Technology, Noordwijk, Netherlands, 2008.

[4] J.-I. Park, H.-E. Park, S.-Y. Park, and K.-H. Choi, "Hardware-in-the-loop simulations of GPS-based navigation and control for satellite formation flying," Adv. Space Res., vol. 46, no. 11, pp. 1451-1465, 2010.

[5] H.-E. Park, S.-Y. Park, S.-W. Kim, and C. Park, "Integrated orbit and attitude hardware-in-the-loop simulations for autonomous satellite formation flying," Adv. Space Res., vol. 52, no. 12, pp. 2052-2066, 2013.

[6] S. Jung, S.-Y. Park, H.-E. Park, C.-D. Park, S.-W. Kim, and Y.-S. Jang, "Real-time determination of relative position between satellites using laser ranging," J. Astron. Space Sci., vol. 29, no. 4, pp. 351-362, 2012.

[7] K. Lee, H. Oh, H.-E. Park, S.-Y. Park, and C. Park, "Laser-based relative navigation using GPS measurements for spacecraft formation flying," J. Astron. Space Sci., vol. 32, no. 4, pp. 387-393, 2015.

[8] H. Oh, H.-E. Park, K. Lee, S.-Y. Park, and C. Park, "Improved GPS-based satellites relative navigation using femtosecond laser relative distance measurements," J. Astron. Space Sci., vol. 33, no. 1, pp. 45-54, 2016.

[9] Y.-S. Jang, K. Lee, S. Han, J. Lee, Y.-J. Kim, and S.-W. Kim, "Absolute distance measurement with extension of nonambiguity range using the frequency comb of a femtosecond laser," Opt. Eng., vol. 53, no. 12, 122403, 2014. 